\title{
Stress analysis of osteoporotic femur
}

\author{
Oleg Ardatov', Vladimir Barsukov ${ }^{2}$, Dmitriy Karev ${ }^{3}$ \\ ${ }^{1}$ Vilnius Gediminas Technical University, Lithuania \\ ${ }^{2}$ Yanka Kupala State University of Grodno, Belarus \\ ${ }^{3}$ Grodno Medical State University, Belarus \\ E-mails: ${ }^{1}$ oleg.ardatov@vgtu.lt (corresponding author), ${ }^{2}$ v.g.barsukov@grsu.by, \\ 3 dmitriy.karev@gmail.com \\ (Received 24 March 2016; accepted 28 April 2016)
}

\begin{abstract}
Osteoporosis and degenerative diseases cause low bone mass that increases fracture risks. This study presents the modelling of osteoporotic femur by employing finite element method (FEM). The loading of femur using FEM tools was performed. The level of degradation was modelled by changing the thickness of cortical shell and using power-law equations, which determine the dependence between apparent density of cancellous bone and its mechanical properties. Obtained results could be useful for both medical diagnosis and bone health check.
\end{abstract}

Keywords: osteoporosis, finite element method, bone mechanics, femur.

\section{Introduction}

Osteoporosis is a disease characterized by low bone mass and micro-architectural deterioration of bone tissue, with a consequent increase in bone fragility and susceptibility to fracture [1]. Osteoporosis is one of the most common health problems affecting both men and women, and it is becoming increasingly prevalent in our aging society. Osteoporosis affects over 200 million people worldwide [2] with an estimated 1.5 million fractures annually in the United States alone [3] and attendant costs exceeding $\$ 10$ billion per annum [4]. Although osteoporosis affects the entire skeleton, many osteoporotic fractures occur in the femur [5]. It has a high mortality rate: survival is $72 \%$ in the first year and only $28 \%$ after five years [5].

Due to the complex anatomy of the femur body, the difficulties associated with obtaining bones for in vitro experiments, and the limitations on the control of the experimental parameters, finite element models have been developed in order to analyse the biomechanical properties of bone tissue.

The present study is aimed to determine the stress distribution on cortical shell of femur with verification of various grades of osteoporosis.

\section{Methods}

A three-dimensional continuum boundary problem is raised. In order to define the mechanical behaviour of femur model under the compression load, theory of elasticity was applied. Main equations are presented in tensor form:

$$
\begin{aligned}
& \frac{\partial \sigma_{i j}}{\partial x_{j}}+f_{i}=0, \\
& \varepsilon_{i j}=\frac{1}{2}\left(\frac{\partial U_{i}}{\partial x_{j}}+\frac{\partial U_{j}}{\partial x_{i}}\right), \\
& \sigma_{i j}=C_{i j k l} \times \varepsilon_{k l},
\end{aligned}
$$

where $\sigma_{i j}$ is a tensor of stress, $\varepsilon_{i j}$ is a strain tensor, $U$ is a displacement tensor, $f_{i}$ corresponds to volume forces and $C_{i j k l}$ is a fourth-order tensor of elasticity. 
The von Mises-Hencky criterion is chosen to analyse the stressed state of the model. The selection of this criterion is based on mechanical properties of the bone, which seems to behave as a ductile material [6]. Also, the model is continuous and isotropic, so the von Mises stress criterion is applied to the research of stresses, which occur on cortical shell of the model.

The von Mises stress is defined in Eq. (4) below, where $\sigma_{1}, \sigma_{2}$ and $\sigma_{3}$ are the maximum, intermediate, and minimum principal stresses respectively; $\sigma_{e q}$ is a von Mises equivalent stress:

$$
\sqrt{\frac{\left(\sigma_{1}-\sigma_{2}\right)^{2}+\left(\sigma_{2}-\sigma_{3}\right)^{2}+\left(\sigma_{3}-\sigma_{1}\right)^{2}}{2}}=\sigma_{e q}
$$

The initial geometry of the model was derived from DICOM data files and converted into the numerical body using SolidWorks software. The model consists of two basic components cortical shell and cancellous bone, both modelled as isotropic and elastic. Section view of the model is presented in Fig. 1. Elasticity constants of healthy model were calculated using powerlaw equations [7]. They are presented in Table 1.

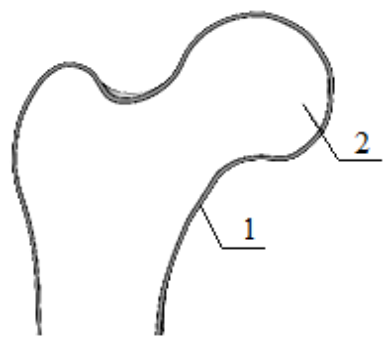

Fig. 1. Section view of numerical femur model: 1 - cortical shell; 2 - cancellous bone

Table 1. Elasticity constants of the healthy model

\begin{tabular}{|c|c|c|}
\hline Component of the model & $E, \mathrm{MPa}$ & $v$ \\
\hline Cortical shell & 18000 & 0.300 \\
\hline Cancellous bone & 3260 & 0.200 \\
\hline
\end{tabular}

The impact of osteoporosis is modelled by decreasing the elasticity modulus of the cancellous bone and thickness of cortical shell, as it is pointed out in Table 2.

Table 2. Varying of parameters of the model

\begin{tabular}{|c|c|}
\hline Parameters of the osteoporotic model & Range \\
\hline Cortical shell thickness, $\mathrm{mm}$ & $0.5-1.5$ \\
\hline Elasticity modulus of cancellous bone & $260-3260$ \\
\hline
\end{tabular}

The model was rigidly constrained through distal part of femur, and static compressive load collinear to axis of femoral neck in the range of 1000-2000 N was applied. The meshing of the model was performed with volume finite elements due to its curvature. The number of elements and nodes is presented in Table 3.

Table 3. Finite element model

\begin{tabular}{|c|c|c|}
\hline Model component & Number of finite elements & Number of nodes \\
\hline Cortical shell & 4157 & 7351 \\
\hline Cancellous bone & 20560 & 45139 \\
\hline
\end{tabular}




\section{Results}

The stress distribution on the cortical shell of the model was obtained for various grades of osteoporotic degradation and model with $0.5 \mathrm{~mm}$ thickness of cortical shell is presented in Fig. 2 .

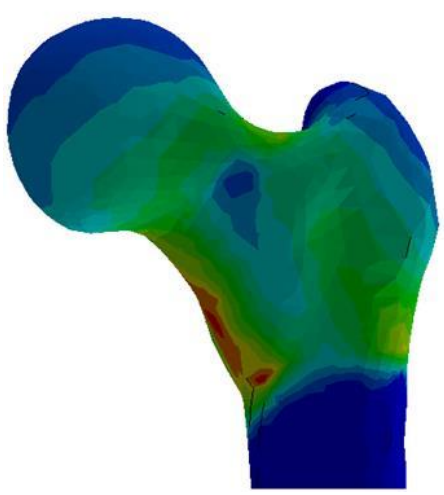

a

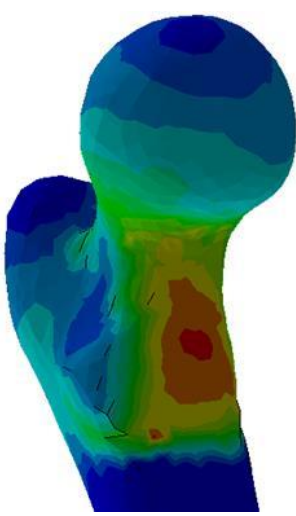

b

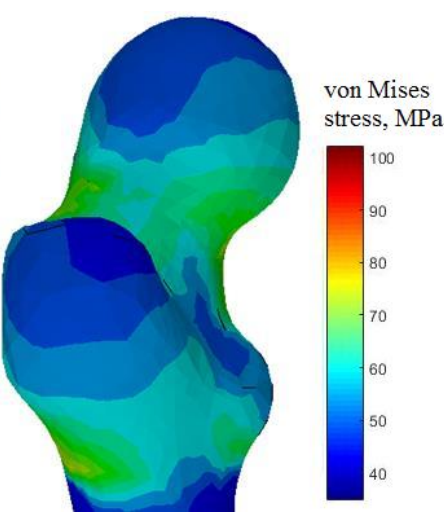

$\mathrm{c}$

Fig. 2. Stress distribution on the cortical shell of femur model with $0.5 \mathrm{~mm}$ cortical shell

As it shown in Fig. 2, the highest von Mises stress appears along the inner area of the neck, near the intertrochanteric line. The difference between the maximal values of stresses in the healthy and osteoporotic model (with $0.5 \mathrm{~mm}$ cortical shell thickness) reaches $350 \%$.

\section{Conclusions}

We developed the finite element model of human femur, which consisted of the cortical shell and cancellous bone. The model was treated for various grades of degenerative diseases. The distribution of von Mises stress was obtained on the cortical shell of the model. It was found, that value of von Mises stress depends both on quality of cancellous bone and thickness of cortical shell, while the thickness of cortical shell remains critical.

\section{References}

[1] Anon. 1993. Consensus development conference: Diagnosis, prophylaxis and treatment of osteoporosis, American Journal of Medicine 94(6): 50-56.

http://dx.doi.org/10.1016/0002-9343(93)90218-E

[2] Lin, J. T.; Lane, J. M. 2004. Osteoporosis: a review, Clinical Orthopaedics and Related Research 425: 34-42. http://dx.doi.org/10.1097/01.blo.0000132404.30139.f2

[3] Nevitt, M. C., et al. 1999. Association of prevalent vertebral fractures, bone density, and alendronate treatment with incident vertebral fractures: effect of number and spinal location of fractures, Bone 25(5): 9-13. http://dx.doi.org/10.1016/S8756-3282(99)00202-1

[4] Johnell, O., et al. 2004. Mortality after osteoporotic fractures, Osteoporosis International 15(1): 3842. http://dx.doi.org/10.1007/s00198-003-1490-4

[5] Riggs, B. L.; Melton 3rd, L. J. 1995. The worldwide problem of osteoporosis: Insights afforded by epidemiology, Bone 17(5): 501S-505S. http://dx.doi.org/10.1016/8756-3282(95)00258-4

[6] Doblaré, M.; García, J. M.; Gómez M. J. 2004. Modelling bone tissue fracture and healing: a review, Engineering Fracture Mechanics 71(13-14): 1809-1840. http://dx.doi.org/10.1016/j.engfracmech.2003.08.003

[7] Helgason, B., et al. 2008. Mathematical relationships between bone density and mechanical properties: a literature review, Clinical Biomechanics 23(2): 135-146.

http://dx.doi.org/10.1016/j.clinbiomech.2007.08.024 